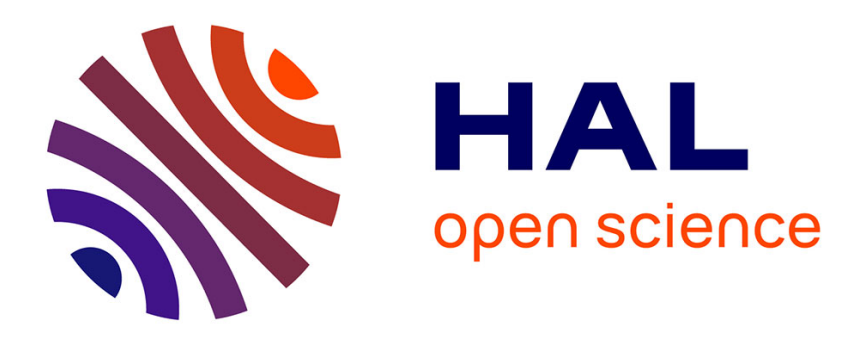

\title{
Jeunes et cultures : reproduction sociale et dynamiques individuelles
}

Pierre Bruno

\section{To cite this version:}

Pierre Bruno. Jeunes et cultures: reproduction sociale et dynamiques individuelles. Le Français Aujourd'hui, 2012, L'attention aux différences, 177, pp.133-141. 10.3917/lfa.177.0133 halshs-01120010

\section{HAL Id: halshs-01120010 \\ https://shs.hal.science/halshs-01120010}

Submitted on 30 May 2017

HAL is a multi-disciplinary open access archive for the deposit and dissemination of scientific research documents, whether they are published or not. The documents may come from teaching and research institutions in France or abroad, or from public or private research centers.
L'archive ouverte pluridisciplinaire HAL, est destinée au dépôt et à la diffusion de documents scientifiques de niveau recherche, publiés ou non, émanant des établissements d'enseignement et de recherche français ou étrangers, des laboratoires publics ou privés. 


\section{JEUNES ET CULTURES : REPRODUCTION SOCIALE ET DYNAMIQUES INDIVIDUELLES}

Pierre BRUNO

Institut universitaire de technologie de Dijon

Ces deux dernières années, le ministère de la Culture a publié de nombreuses enquêtes qui portent un regard nouveau ou actualisé sur les pratiques culturelles des jeunes : mise en perspective des enquêtes de ces quarante dernières années, publication des résultats inédits de l'enquête spécifiquement consacrée aux jeunes, organisation d'un colloque international (Enfance et cultures : regards des sciences humaines et sociales ${ }^{1}$ ). Ces travaux témoignent de l'intérêt porté à ce corpus dans lequel s'inscrivent d'autres initiatives de moindre ampleur mais tout aussi significatives (Cahiers pédagogiques de janvier 2011 consacré à " Culture de l'école, cultures des jeunes " (voir notre compte rendu infra), forum de Télérama des 6 et 7 avril 2012 « Nos enfants et la culture »...).

\section{Approches macrosociologiques et microsociologiques}

En 2011, Le ministère de la Culture publie deux synthèses de ses enquêtes sur les pratiques culturelles des Français ${ }^{2}$, l'une cherchant à en dégager les grandes lignes de force ${ }^{3}$, l'autre abordant les questions méthodologiques ${ }^{4}$. Si elles ne traitent que partiellement des publics jeunes (et plus précisément des 15 ans et plus), ces études ont un double intérêt. O. Donnat a tout d'abord le mérite de dénoncer « les facilités des discours sur la "révolution numérique" qui, faute d'une réelle mise en perspective historique, tendent à présenter comme des ruptures radicales des évolutions dont l'origine est parfois bien antérieure à l'arrivée de l'internet " 5 . De même il faut lui reconnaitre aussi le mérite de reconnaitre l'importance des nouvelles formes d'inégalités et plus particulièrement de :

1. Les actes sont disponibles en ligne : <www.enfanceetcultures.culture.gouv.fr>

2. Ces travaux sont disponibles à l'adresse suivante : <www.pratiquesculturelles.culture. gouv.fr>

3. O. Donnat, Pratiques culturelles, 1973-2008. Dynamiques générationnelles et pesanteurs sociales, Paris : Ministère de la Culture et de la Communication, DEPS, coll. "Culture études ", 2011-7, décembre 2011.

4. O. Donnat, Pratiques culturelles, 1973-2008. Questions de mesure et d'interprétation des résultats, avec la collaboration de Florence Lévy, Paris : Ministère de la Culture et de la Communication, DEPS, coll. "Culture méthodes », 2011-2, décembre 2011.

5. Dynamiques générationnelles, Ibid., $2011: 1$. 
la question des inégalités territoriales [qui] se pose dans des termes analogues à celle des inégalités sociales. Quand on s'interroge sur les variations des écarts entre ruraux et urbains (ou entre les Parisiens et les autres Français), il faut prendre en compte les transformations des différentes populations concernées (élitisation de la population parisienne, par exemple, liée à la flambée des prix de l'immobilier, rurbanisation de certaines communes rurales, etc.). ${ }^{6}$

\section{Ces synthèses contribuent ainsi au nécessaire rappel :}

- une fois encore, diront certains - [de] la permanence de profonds clivages sociaux et territoriaux en matière de lecture de livres ou de fréquentation régulière des équipements culturels [qui] peut apparaitre inutile ou redondant. Pourtant, comment ne pas le faire au moment où bon nombre d'observateurs (et de sociologues) abordent la question de l'individualisme contemporain en " oubliant " de situer socialement les individus dont ils analysent les comportements, comme si le processus d'individualisation était socialement indifférencié, alors qu'il repose sur des supports et des ressources tant matérielles que cognitives inégalement réparties dans notre société. ${ }^{7}$

Dans son approche de la jeunesse, O. Donnat se démarque d'un discours sociologique ou anthropologique qui tend à analyser cette population en termes d'âge, de dispositions spécifiques ou de valeurs, pour lui substituer une vision qui en differe sur trois points.

1. La jeunesse se caractérise moins par des effets d'âge (liés aux conditions de vie spécifiques par exemple) que par des effets de génération :

L'évolution des pratiques culturelles doit-elle être appréciée d'un double point de vue difficilement conciliable : le premier souligne la permanence d'une forte stratification sociale des pratiques culturelles et confirme la pertinence des schémas théoriques articulés autour de la notion de capital culturel, tandis que le second met en lumière la force des mutations générationnelles, rappelant que les formes de la domination culturelle, loin d'être éternelles, se renouvèlent en liaison avec les transformations de la structure sociale, des conditions d'accès à la culture et des modes d'expression artistique. Aucune de ces deux perspectives ne doit être privilégiée au détriment de l'autre. (Ibid. : 28)

2. Cet âge de la vie ne se distingue par des dispositions spécifiques (soif d'absolu, mutations physiologiques...) mais par des conditions de vie spécifiques (temps libre, sociabilité, revenus...) :

D'autres éléments sont à prendre en compte, à commencer par le fait que de plus en plus d'adultes retrouvent, parfois à un âge avancé, des conditions de vie proches de celles de la postadolescence du fait des transformations de la vie de couple et de l'augmentation du nombre de séparations : célibataires, divorcés, personnes en couple vivant séparément, etc., autant de situations qui, aujourd'hui, peuvent conduire à prolonger ou à renouer avec un mode de loisir juvénile, avec des niveaux élevés de sociabilité amicale ou de sorties culturelles. (Ibid. : 33)

6. Questions de mesure, Ibid. : 9.

7. Dynamiques générationnelles, Ibid. : 28. 
3. Si l'on a longtemps célébré les valeurs prêtées à la jeunesse, il faut inverser les termes de la problématique et penser la jeunesse comme valeur :

La cote de la jeunesse n'a cessé de grimper à la bourse des valeurs depuis les années 1970. [...] Le fait d'être ou de paraitre jeune a cessé, dans notre société, d'être une simple question d'âge pour devenir une finalité en soi que les images publicitaires nous rappellent en permanence. Cette injonction récurrente à présenter les signes extérieurs de la jeunesse se traduit en termes de consommation ou de mode de vie par le succès des marchés de la mode, du sport, de la forme ou de la chirurgie esthétique, mais aussi par l'évolution des industries culturelles qui, tout en conservant les adolescents comme cible privilégiée, ont de plus en plus tendance à jouer la logique générationnelle en proposant des produits renforçant les liens qui relient les adultes à leur "vie d'avant " : stations de radio et chaines de télévision générationnelles, concerts de groupes rock des années 1970 ou 1980, rediffusion de séries et d'émissions télévisées rétro, mode des rétrogames dans le domaine des jeux vidéo, etc. (Ibid. : 33)

Cette mise en perspective des enquêtes réalisées entre 1973 et 2008 se fait au moment même de la publication de deux ouvrages consacrés plus spécifiquement au rapport des enfants et des adolescents à la culture ${ }^{8}$. L'Enfance des loisirs est le résultat d'une enquête qui s'inscrit dans une logique différente mais complémentaire des travaux précédents.

Au début des années 2000, le ministère de la Culture et de la Communication constate à la fois l'« éternel retour du même : progressions millimétriques voire nulles, permanence des mêmes hiérarchies et des mêmes écarts, réitération des mêmes disparités sociales ou géographiques " et, par ailleurs, "la diversité des usages, des gouts et des itinéraires culturels ainsi que le jeu complexe des "petits" facteurs explicatifs non identifiables à l'échelle de la population française $"{ }^{9}$. Pour tenter de concilier l'étude des masses et celle des parcours individuels, l'enquête menée auprès des jeunes substitue à l'approche synchronique de la Distinction ou des enquêtes publiées depuis 1990, une approche plus ambitieuse, plus lourde à mettre en place, qui suit sur une décennie une même cohorte de jeunes afin de mesurer le poids des contraintes collectives et celui des stratégies individuelles. Cette démarche se subdivise en quatre temps qui sont autant de parties de l'ouvrage : une analyse dynamique de l'enfance comme avancée dans l'âge (Chap. 1) marquée par l'influence du genre et de l'origine sociale (Chap 2) et par une pluralité des transmissions (famille, pairs, école... Chap 3) où la culture permet, par le jeu des trajectoires communes et des parcours individuels, la création de l'identité personnelle (Chap 4).

Comme on peut le lire dans le quatrième de couverture de L'Enfance des loisirs, ces études cherchent donc à dégager une approche nouvelle de

8. S. Octobre, C. Détrez, P. Mercklé et N. Berthomier, L'Enfance des loisirs. Trajectoires communes et parcours individuels de la fin de l'enfance à la grande adolescence, Paris : Ministère de la Culture et de la Communication, La Documentation française, coll. "Questions de culture ", 2010 ; S. Octobre (dir.), Enfance \& culture. Transmission, appropriation et représentation, La Documentation française, coll. « questions de culture », 2010.

9. O. Donnat et P. Tolila, Présentation, in O. Donnat et P. Tolila (dir.), Le(s) Public(s) de la culture, Paris : Presses de Sciences Po, 2003, pp. 17-18. 
l'enfance qui se démarquerait d'une vision qui les a « longtemps considérés comme des "héritiers" reproduisant les comportements parentaux » et d'un courant qui les décrit " comme des consommateurs passifs soumis à l'offre médiatico-publicitaire des industries ". L'idée est donc de dépasser un antagonisme que l'on pourrait juger factice ou stérile dans la mesure où les deux points de vue partageraient une même vision passive de l'enfance, alors que ces travaux mettent, eux, en exergue un "métier de consommateur culturel à la croisée des autres métiers de l'enfance ${ }^{10}$ et valorisent :

la capacité d'action de l'enfant sur son environnement, son travail sur soi et sur le monde social qui l'entoure, les compétences, savoir, savoir faire et savoir être qu'il développe, de même que le jeu subtil des contraintes dans lequel il est pris. Le concept d'agency le dit plus largement encore en anglais, empruntant autant à la sociologie interactionniste symbolique qu'aux théories constructivistes. [...] Au concept de reproduction se substitue celui de " reproduction interprétative " pour dire ce travail de l'enfant sur le monde social et permettre de penser les transformations et dynamiques générationnelles. (Octobre $2001: 11$ )

\section{Individualisation et culturalisation des inégalités?}

Les deux perspectives ministérielles s'avèrent donc complémentaires. Au risque de caricaturer les positions des uns et des autres, on pourrait dire que les synthèses d'O. Donnat insistent sur l'analyse des " pesanteurs sociales ", là où les travaux pilotés par $S$. Octobre privilégient la genèse de "dynamiques générationnelles ", étudiées ici sous l'angle de la construction de soi par des individus actifs. Face à ce qui pourrait même apparaitre comme un constat d'échec de la politique de démocratisation culturelle du ministère, on pourrait y voir, au risque d'extrapolation hasardeuses, de nouvelles pistes d'action qui privilégieraient les apprentissages précoces et chercheraient à agir sur les dynamiques individuelles.

Lorsqu'on observe les conditions qui ont permis l'émergence et la construction de cet objet scientifique spécifique (sur ce point le lecteur pourra se reporter à l'étude de R. Sirota ${ }^{11}$ ), cette sociologie des pratiques culturelles juvéniles se ressent d'influences plus larges. L'objectivation de ces variables ne doit pas être instrumentalisée pour remettre en cause ces travaux et leurs résultats, mais peut aider à en comprendre certains présupposés et donc, peut être, certaines limites. Tout d'abord cette sociologie s'inscrit dans un large mouvement de renouveau, au sein des sciences humaines, d'approches plus individualistes (ou plus précisément « anthropologiques ») en rupture avec la prégnance du paradigme bourdieusien de la culture par rapport auquel les travaux à venir chercheront toujours à se positionner (pour le contester, l'actualiser, etc.). On observe aussi l'influ-

10. S. Octobre et N. Berthomier, L'Enfance des loisirs Éléments de synthèse, coll. "Culture études ", 2011-6 <http://www.culturecommunication.gouv.fr/Politiques-ministerielles/ Etudes-et-statistiques/Articles/L-enfance-des-loisirs>; S. Octobre, Présentation. "Le Genre, la culture et l'enfance ", Réseaux, 4/2011, 168-169, 9-22.

11. R. Sirota, De l'indifférence sociologique à la difficile reconnaissance de l'effervescence culturelle d'une classe d'âge, Enfance et culture, Ibid., pp. 19-38. 
ence d'approches anglosaxonnes (cultural studies, youth studies...), souvent définies par leur objet et jugées mieux armées conceptuellement pour la mesure de cet objet d'étude. Évolutions concordantes dont R. Sirota pointe bien les enjeux politiques (en termes de philosophie politique) :

On ne peut cependant pas exclure de la construction de ce nouveau regard scientifique, la réflexion issue de la philosophie politique qui réexamine la crise de la transmission, qu'elle soit scolaire, familiale ou culturelle. Car c'est bien aussi dans une discussion idéologique que s'inscrivent nombre de travaux sociologiques déplorant tyrannie de la majorité ou mettant à l'honneur la culture de la chambre. Elles s'interrogent tant sur le nouveau statut de l'enfant que sur les modes de transmissions et leurs régimes d'autorité, la négociation semblant en être devenue l'une des figures majeures, transcendant l'échiquier des classes sociales. (Ibid. : 37)

Par ailleurs, même si des études précises restent à faire pour ce corpus précis, cette évolution des perspectives recoupe celle des conditions même de production de savoir comme l'avait montré A. Berger ${ }^{12}$ pour les gender studies dans un numéro précédent du Français aujourd'hui:

Comment interpréter ces nouveaux signes de bienveillance, voire d'intérêt pour notre champ, envoyés par les instances décisionnelles de l'institution universitaire, et même par les commissaires de l'État? Je ne crois pas, en tout cas pas simplement, à une révolution des esprits. Il me semble que cet infléchissement, encore timide et incertain, est surtout le résultat de la convergence de deux facteurs : la substitution, dans l'enseignement supérieur, d'une logique de compétition internationale aux principes qui gouvernaient jusque-là l'ensemble du système éducatif français, et le renversement de la position de la France intellectuelle qui, d'exportatrice majeure d'idées dans le monde, est devenue depuis quelques années une importatrice avide des discours les plus " en vue » hors de ses frontières. (Berger, ibid. : 88)

Cette double influence n'implique pas forcément que ces approches soient incompatibles avec le modèle bourdieusien, même si certaines études en dénoncent la "simplicité " (censée en expliquer le succès) ${ }^{13}$ et si cette dimension est absente de travaux comme celui sur les jeux vidéos ${ }^{14}$ où le gout se constitue essentiellement au sein de la fratrie, alors que d'autres travaux plus anciens avaient pu montrer aussi l'existence de pratiques plus ou moins distinctives de ce loisir. Contrairement aux travaux de D. Pasquier ou de M. Petit, L'Enfance des loisirs ne se caractérise ni par un rejet (ou un dépassement) marqué du modèle bourdieusien ni par une négation des inégalités sociales ou des logiques contribuant à la reproduction des hiérarchies existantes :

12. A.E. Berger, 2008, Petite histoire paradoxale des études dites de "genre » en France, Le Français aujourd'hui, 163, 83-91.

13. J. Zaffran et M.-L. Pouchadon, La recomposition des pratiques culturelles des adolescent(e)s : Terrain français, éclairages québécois, Enfance et culture, p. 171.

14. F. Dajez et N. Roucous, Le jeu vidéo, une affaire d'enfants. Enquête sur le parc à jouets numérique d'enfants de 6 à 11 ans, Enfance et culture, pp. 85-101. 
Les métiers envisagés pour l'avenir témoignent également de l'intériorisation des possibles et de son évolution avec l'avancée en âge [...] les filles d'ouvriers se rêvent coiffeuse, docteur ou infirmière ou enseignante, ces deux dernières professions les rapprochant des filles de cadre qui aimeraient aussi être scientifique, avocate, architecte, journaliste ou photographe... ${ }^{15}$

La dimension distinctive de la culture est parfois rappelée, comme dans la constitution des gouts musicaux :

Les écarts entre les gouts des enfants de cadres et des enfants d'ouvriers se creusent, le jazz, et encore plus clairement le rock, devenant des genres musicaux très marqués socialement (à 17 ans, les enfants de cadre ont désormais quatre fois plus de chance d'apprécier le rock que les enfants d'ouvriers), tandis que le rap, le R'n'B et dans une moindre mesure le hip hop deviennent des genres musicaux populaires. ${ }^{16}$

Afin de compenser le capital négatif que représentent les gouts féminins, les filles de cadres tendraient à rejeter les préférences des filles de milieu populaire (dont le R'n'B serait représentatif) en adoptant le gout des garçons de leur milieu social. ${ }^{17}$

Toutefois, et c'est là que porte le fond de la différence, ces inégalités apparaissent désormais comme une variable et non plus comme une logique. Elles sont un obstacle à surmonter et non plus une finalité à contester. Cette modification de perspective générale se retrouve dans la définition des fonctions prêtées à la culture. Dans une logique de "culturalisation des identités " ${ }^{18}$ celle-ci se voit attribuer le rôle de formation de l'individu : « les pratiques culturelles et de loisirs disent donc qui on est : elles contribuent à façonner l'identité aussi bien dans les mises en jeu de soi, corporellement et symboliquement, que dans les imaginaires " ${ }^{19}$. Qu'il s'agisse de "rendre à la dimension individuelle toute son épaisseur et son irréductibilité ${ }^{20}$ ou de valoriser "l'infinie diversité des façons de grandir et de tracer son chemin " ${ }^{21}$ comme les "braconnages juvéniles " qui permettent d' "être soi en métissant les influences " ${ }^{22}$, L'Enfance des loisirs se différencie d'une approche structurale et critique de la culture moins par son attachement premier à la construction de l'individu, mais par le fait que ce dernier est avant tout défini par son identité, sa singularité et non pas par sa position au sein d'une structure ou d'un champ. Cette orientation explique que les contraintes externes apparaissent comme minorées sur au moins trois points :

15. L'Enfance des loisirs, p. 151.

16. Ibid., p. 140.

17. Ibid., p. 141.

18. S. Octobre et N. Berthomier, L'Enfance des loisirs. Éléments de synthèse, coll. "Culture études ", 2011-6 <http://www.culturecommunication.gouv.fr/Politiques-ministerielles/ Etudes-et-statistiques/Articles/L-enfance-des-loisirs>

19. L'Enfance des loisirs, p. 151.

20. Ibid., p. 21.

21. Ibid., p. 327.

22. Enfance et culture, p. 218. 
1) L'étude reconnait son absence de prise en compte de l'offre et s'interroge sur :

la nécessité d'un questionnement de la production disponible : bien souvent formatée pour correspondre commercialement à ses besoins en termes d'identification de genre, elle n'offre que rarement les outils pour une appropriation dépassant le jeu des stéréotypes pour celles et ceux qui y puisent quasi exclusivement. ${ }^{23}$

Pour autant, les rares études qui prennent en compte ces biens culturels ne traitent qu'incidemment des différences internes aux industries culturelles ${ }^{24}$ qui pourraient, pour d'autres, constituer l'entrée principale de ces corpus.

2) De même, contrairement aux écrits d'O. Donnat, ces travaux n'évoquent que rarement la question des inégalités territoriales ${ }^{25}$ dont les résultats des dernières élections présidentielles ont témoigné de l'importance qu'elles prennent dans la perception des inégalités sociales au quotidien.

3) Les inégalités scolaires, qui avaient pourtant été très bien synthétisées dans un ouvrage précédent du ministère ${ }^{26}$, n’apparaissent pas ou si peu : le système éducatif est un lieu de socialisation plus que d'orientation (voire de relégation). Et nous ne parlerons que, pour mémoire, de l'absence des inégalités des futures positions sociales qui leur est fortement corrélée.

Notons que ces orientations révèlent une rupture fondamentale avec les approches précédentes. L'objet du travail scientifique n'est plus de témoigner - par delà l'apparente diversité des trajectoires individuelles - de l'existence de régularités objectives dont la méconnaissance influe sur le destin de chacun en contribuant à renforcer l'ordre établi, "naturel ", des choses. L'étude tend à montrer au contraire comment l'individu parvient à se construire malgré les inégalités et discriminations potentielles. S'il s'agit ainsi de comprendre la perception commune du monde et non plus de la dépasser, cela n'implique pas forcément une gestion politique libérale de ces cultures. La question du contenu des œuvres conclut aux carences potentielles du seul marché et, nous l'avons vu, à « la nécessité d'un questionnement de la production disponible ». De même, se dessine, comme en filigrane, une nécessaire intervention (de l'État ou pas) pour corriger les inégalités que laisse deviner de manière implicite la typologie des pratiques. Contrairement aux profils dégagés dans l'enquête de J.-M. Guy ${ }^{27}$ et fondés

23. L'Enfance des loisirs, p. 152.

24. C. Peter, Petites princesses contre super-héros : les médias destinés aux 2-14 ans mettentils en scène le clivage des genres ?, Enfance et culture, pp. 129-146.

25. C. Guilluy et C. Noyé, Atlas des nouvelles fractures sociales : les classes moyennes oubliées et précarisées, Paris : Autrement, 2004.

26. F. Dubet, Paradoxes et enjeux de l'école de masse, In O. Donnat et P. Tolila (dir.), Les Publics de la culture, Paris : Presses de Sciences Po, 2003.

27. J.-M. Guy, Les Jeunes et les sorties culturelles: fréquentation et image des lieux de spectacles et de patrimoine dans la population française âgée de 12 à 25 ans, Paris : Ministère de la Culture, Département des études et de la prospective, 1995. 
sur des postures divergentes, hiérarchisées, conflictuelles (l'éclectisme, la rébellion, la frustration...) dans l'esprit des profils dégagés par l'enquête de 1989, la définition des parcours est présentée comme un continuum doté d'un caractère normatif :

Cinq catégories principales de trajectoires culturelles, toutes activités confondues, émergent.

- Une trajectoire "très favorable ", cumule loisirs sous toutes ses formes et témoigne d'un investissement précoce, important et durable ( $16 \%$ des enfants, surtout des filles, issus de familles de milieu favorisé dotées en capitaux culturels).

- Une trajectoire «favorable », marquée par un investissement polymorphe malgré la baisse de la lecture (groupe rassemblant $27 \%$ des enfants, plutôt féminin, composé de bons élèves issus de milieu favorisé et intermédiaire).

- Une trajectoire " intermédiaire ", marquée par un investissement durablement modéré, exception faite pour la télévision ( $27 \%$ des enfants, dont la majorité de garçons, et issus le plus souvent de parents titulaires d'un CAP et employés).

- Une trajectoire "défavorable», marquée par les retraits et abandons (21\% des enfants, dont la majorité de garçons, et davantage de fils d'ouvriers que dans la moyenne).

- Une trajectoire " très défavorable ", marquée par l'absence de loisirs et pratiques culturelles (9\% des enfants, ce groupe étant le plus masculin, davantage issus de parents et de grands-parents non diplômés, ouvriers ou inactifs). ${ }^{28}$

Même si cela n'est jamais clairement problématisé dans la composition de l'ouvrage, on devine dans bien des pages la vision d'inégalités liées aux carences éducatives de certains milieux peu "favorables " à l'épanouissement de l'identité de leurs enfants :

les enfants de famille populaire sont davantage et plus précocement équipés en propre de certains matériels de consommation culturelle; notamment médiatiques. [...] Et de même, les contrôles sur les consommations médiatiques sont plus nombreux dans les familles des catégories supérieures, où l'enfant reste plus durablement « sous surveillance ». ${ }^{29}$

Analyse des pesanteurs sociales ou des dynamiques individuelles, les deux approches des cultures de la jeunesse sont plus complémentaires que contradictoires, et correspondent aux deux perspectives définies au début des années 2000 par le ministère de la Culture. Pour autant, et pour

28. P. Mercklé, S. Octobre, C. Détrez et N. Berthomier, Une enquête inédite, Sciences humaines, 226, mai 2011. Consultable en ligne <http://www.scienceshumaines.com/uneenquete-inedite_fr_27072.html> Voir aussi L'Enfance des loisirs, p. 261 et sq.

29. S. Octobre, Les transmissions culturelles chez les adolescents : influences et stratégies individuelles, Enfance et culture, Paris : La documentation française, 2010, p. 211. 
reprendre l'analyse de F. Dubet dans Les Places et les chances ${ }^{30}$ (sur laquelle nous reviendrons dans une prochaine chronique), la sociologie des cultures enfantines et adolescentes s'inscrit dans la tendance actuelle à l'individualisation et à la culturalisation des inégalités sociales. Un modèle où la société n'est plus définie comme un ordre extérieur aux acteurs mais comme une création des individus, un modèle où chacun est défini par une identité culturelle (à respecter) plutôt que par une place dans une hiérarchie (à abolir ou à corriger), un modèle où les différences de statuts choquent moins que l'inégale probabilité d'y accéder selon son " identité " (genre, origine, orientation sexuelle...), un modèle où les difficultés des enfants issus des milieux populaires tiennent pour une part (non négligeable?) à des carences familiales. C'est pourquoi, par delà l'accord sur la qualité et la quantité du travail scientifique fourni, l'évaluation de l'intérêt de l'ouvrage restera marquée par la vision que chacun se fait des inégalités sociales.

Pierre BRUNO 